\title{
The multifunctional human erythrocyte as oxygen transporter and immune and inflammatory modulator in sepsis
}

\author{
Ryon M Bateman PhD
}

\section{INTRODUCTION}

Despite there being over a trillion erythrocytes flowing through the human body, they are generally considered to be rather simple cells-filled with hemoglobin, acting as the circulatory system's primary oxygen carrier. However, evidence suggests that the human red blood cell (RBC) is, in fact, multifunctional ${ }^{1,2}$ and has roles not only in oxygen transport and carbon dioxide removal but also in hypoxic signaling and microvascular blood flow regulation, ${ }^{3}$ chemokine immunomodulation, ${ }^{4}$ inflammation suppression by scavenging mitochondrial DNA $(\mathrm{mtDNA})^{5}$ and bacterial lipopolysaccharide (LPS), ${ }^{6}$ and, possibly, innate immunity. ${ }^{7,8}$

Invading pathogens activate the immune, inflammatory, and coagulation systems. These systems target infection sites but also dramatically alter microvascular environments in remote organs, sequestering RBCs within stopped-flow capillaries, altering microvascular oxygen transport, ${ }^{3}$ and creating local hypoxic microenvironments with the potential to increase oxidative stress and tissue damage. ${ }^{2}$ Pathogens can adhere to RBCs via glycophorin A (GYPA), strip it of sialic acid residues ${ }^{9}$, and alter RBC surface charge. Or they may release proteolytic enzymes, toxins, and virulence factors that can damage intracellular and/or integral RBC membrane proteins, induce hemolysis, or trigger a series of events, including oxygen free radical generation, intracellular $\mathrm{Ca}^{2+}$ accumulation and the flipping of membrane phospholipid, phosphatidylserine (PS) to the outer membrane leaflet ${ }^{10}$ leading to eryptosis, coagulation, and clearance of damaged RBCs by macrophages in the spleen or liver. In addition, activated neutrophils and endothelial cells expose RBCs

Corresponding author: Ryon Bateman

Contact Information: ryon.bateman@gmail.com DOI: 10.12746/swrccc.v7i28.536 to oxygen free radicals and reactive oxygen species, stressing their endogenous antioxidant capacity and oxidizing membrane components. ${ }^{2}$

\section{SEPSIS ALTERS ERYTHROCYTE MORPHOLOGY}

An early indication of sepsis-induced erythrocyte damage may be evident in simple blood smears. Under septic conditions, a fraction of the RBC population undergoes dramatic morphological change, and normal discoid RBCs become crenated (echinocyte) or spherical (spherocyte). ${ }^{2,9}$ These changes are associated with changes in cell volume and indicate impaired volume regulation and/or cytoskeletal damage. Practically, an increase in the red cell distribution width (RDW) parameter (the standard deviation of cell volume over its mean measured during automated blood analysis) is an indication of increased heterogeneity in erythrocyte morphology. Moreover, a blood smear may also reveal the presence of sepsis-induced RBC aggregation.

\section{SEPSIS—INDUCED CHANGES IN ERYTHROCYTE DEFORMABILITY}

The average human RBC is approximately $2 \mu \mathrm{m}$ thick with an $8 \mu \mathrm{m}$ diameter, whereas the average skeletal muscle capillary has a diameter of approximately $5 \mu \mathrm{m}$, and splenic slits have narrower $2 \mu \mathrm{m}$ openings. Accordingly, erythrocytes must dramatically deform to pass through the spleen and bend around numerous curves and bifurcations in capillary networks. Panel $A$ in Figure 1 shows RBCs deforming as they flow through a capillary. In both septic patients and animal models, sepsis has been found to decrease erythrocyte deformability (RBCD).

Animal models have found that RBCD decreases progressively over the first 3-6 hours following induction of fecal peritonitis, is associated with a subpopulation of $\mathrm{RBCs}$ and oxidative membrane damage, and is linked 


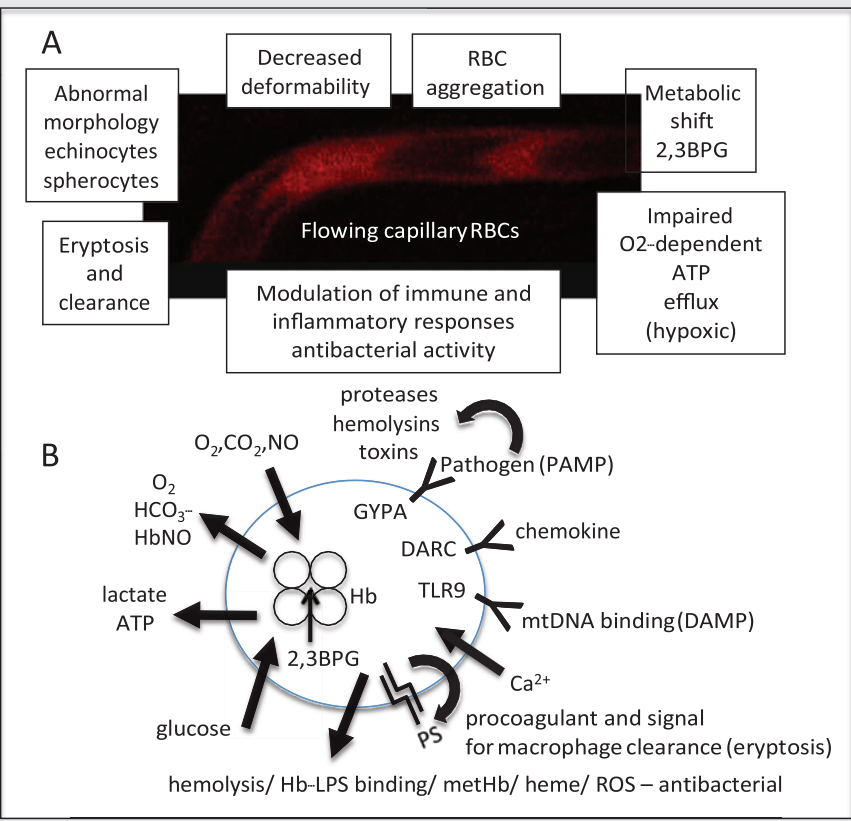

Figure 1. The erythrocyte as an oxygen transporter and a modulator of immune and inflammatory signals in sepsis.

Panel A shows flowing RBCs in a capillary. Deformed RBCs are dark against the red contrast medium. Sepsis alters RBC morphology, deformability, aggregation, metabolism and function. The diagram in panel B shows gases transported by the $\mathrm{RBC}\left(\mathrm{O}_{2}, \mathrm{CO}_{2}, \mathrm{NO}\right)$, surface receptors, GYPA, DARC and TLR9, and the phospholipid PS (described in text). The RBC uses glycolysis to generate ATP that can also be used in hypoxic signaling, as well as the $\mathrm{Hb}$ allosteric regulator 2,3BPG. Damaged RBCs may undergo eryptosis and be cleared by the liver or spleen. Hemolyzed RBCs exhibit antibacterial killing properties. $\mathrm{Hb}$ - hemoglobin, $\mathrm{HbNO}$ - NO bound to $\mathrm{Hb}$, DAMP (danger-associated molecular pattern), PAMP (pathogen-associated molecular pattern), ROS (reactive oxygen species).

with cellular events leading to eryptosis and clearance, ${ }^{3,10,11}$ suggesting that decreased RBCD may facilitate capture of damaged RBCs by the spleen or liver. Whether less deformable RBCs are the principal factor responsible for the loss of functional capillary density in the septic muscle microcirculation is unknown.

\section{METABOLIC SHIFTS, ALTERED HEMOGLOBIN OXYGEN AFFINITY AND IMPAIRED RBC HYPOXIC SIGNALING}

Human erythrocytes lack mitochondria. Consequently, the RBC relies on glycolysis to generate ATP. Moreover, its glycolytic pathway has a unique sidebranch (The Rapoport-Lubering shuttle) that generates 2,3 bisphosphoglycerate (2,3BPG, an allosteric regulator of hemoglobin) under hypoxic conditions. 2,3BPG decreases $\mathrm{Hb}$ affinity for oxygen, promoting oxygen release and shifting the oxygen dissociation curve to the right. In animal models, LPS increased 2,3BPG and decreased intracellular ATP in mice but decreased 2,3BPG in baboons. In septic shock patients, 2,3BPG correlated with arterial $\mathrm{pH}$, suggesting that hemoglobin's affinity for oxygen was altered by acidosis. ${ }^{2,12}$

In addition, evidence suggests the RBC can signal the microvascular network to increase blood flow to hypoxic regions via an $\mathrm{RBC} \mathrm{O}_{2}$-dependent ATP efflux, hypoxic signaling pathway. ${ }^{13}$ Here, deoxyhemoglobin displaces glycolytic enzymes bound to the inner cell membrane, facilitating increased ATP production and ATP efflux. ATP then diffuses to adjacent endothelial cells, triggering a conducted response back through the capillary network to upstream arterioles, causing them to dilate and increase downstream blood flow. Of potential significance in septic patients is the finding that $\mathrm{RBC} \mathrm{O}_{2}$-dependent ATP efflux (hypoxic signaling) is impaired in septic rats, ${ }^{3}$ consistent with a loss of microvascular autoregulation.

\section{ERYTHROCYTE MODULATION OF IMMUNE AND INFLAMMATORY RESPONSES AND POSSIBLE ROLE IN INNATE IMMUNITY}

Human erythrocytes also lack a nucleus and ribosomes and are thus unable to upregulate and synthesize de novo proteins in response to sepsis-induced pro-inflammatory cytokines. However, the RBC can modulate the immune response via the Duffy $A G$ receptor (DARC) which binds chemokines responsible for neutrophil migration. ${ }^{4}$ In addition, the RBC can also bind mtDNA by the TLR 9 receptor $^{5}$ and, thereby, remove a pro-inflammatory stimulus from 
the septic environment. This interaction causes the $\mathrm{RBC}$ to crenate, raising the possibility that the affected $\mathrm{RBC}$ will ultimately undergo eryptosis and be cleared from the circulation.

And while bacteria can adhere and invade RBCs by binding with GYPA causing severe cellular damage, it has been suggested that GYPA may also act as a "decoy receptor"7 preventing infection of tissue. ${ }^{1}$ Somewhat paradoxically, hemolysis may result in a form of innate immunity as free hemoglobin subunits can bind LPS, ${ }^{6}$ and both methemoglobin and free heme can generate sufficient reactive oxygen species to kill invading pathogens. ${ }^{8}$ Though, the presence of free heme is lethal in some models. ${ }^{14}$

\section{SUMMARY}

The human erythrocyte is a multifunctional blood cell. During sepsis, the RBC is altered morphologically (shape), biophysically (RBCD), metabolically (2,3BPG), and functionally (impaired hypoxic signaling). However, the RBC does have the capacity to modulate both immune (chemokine) and inflammatory (mtDNA) signals and the ability to kill pathogens via hemoglobin generated ROS. The large number of RBCs also provides a degree of protection against septic injury, provided the infection does not overwhelm the host.

Keywords: sepsis, erythrocytes, morphology, inflammation

Article citation: Bateman RM. The multifunctional human erythrocyte as oxygen transporter and immune and inflammatory modulator in sepsis. The Southwest Respiratory and Critical Care Chronicles 2019;7(28):1-3

From: Department of Medical Biophysics, University of Western Ontario, London, Ontario, Canada

Submitted: $3 / 16 / 2019$

Accepted: $4 / 2 / 2019$

Conflicts of interest: none

This work is licensed under a Creative Commons Attribution-ShareAlike 4.0 International License.

\section{REFERENCES}

1. Anderson HL, Brodsky IE, Mangalmurti NS. The evolving erythrocyte: red blood cells as modulators of innate immunity. J Immunol 2018;201(5):1343-1351.

2. Bateman RM, Sharpe MD, Singer M, et al. The effect of sepsis on the erythrocyte. Int J Mol Sci 2017;18(9).

3. Bateman RM, Sharpe MD, Jagger JE, et al. Sepsis impairs microvascular autoregulation and delays capillary response within hypoxic capillaries. Crit Care 2015;19:389.

4. Lee JS, Wurfel MM, Matute-Bello G, et al. The Duffy antigen modifies systemic and local tissue chemokine responses following lipopolysaccharide stimulation. J Immunol 2006; 177:8086-8094.

5. Hotz MJ, Qing D, Shashaty MGS, et al. Red blood cells homeostatically bind mitochondrial DNA through TLR9 to maintain quiescence and to prevent lung injury. Am J Respir Crit Care Med 2018;197(4):470-480.

6. Bahl N, Du R, Winarsih I, Ho B, et al. Delineation of lipopolysaccharide (LPS) - binding sites on hemoglobin: from in silico predictions to biophysical characterization. $J$ Biol Chem 2011;286(43):37793-37803.

7. Baum J, Ward RH, Conway DJ: Natural selection on the erythrocyte surface. Mol Biol Evol 2002;19(3):223-229.

8. Jiang N, Tan NS, Ho B, et al. Respiratory protein - generated reactive oxygen species as an antimicrobial strategy. Nat Immunol 2007;8(10):1114-1122.

9. Piagnerelli M, Boudjeltia KZ, Rapotec A, et al. Neuraminidase alters red blood cells in sepsis. Crit Care Med 2009; 37(4):1244-1250.

10. Qadri SM, Donkor DA, Bhakta V, et al. Phosphatidylserine externalization and procoagulant activation of erythrocytes induced by Pseudomonas aeruginosa virulence factor pyocyanin. J Cell Mol Med 2016;20(4):710-720.

11. BatemanRM,JaggerJE,SharpeMD, etal.Erythrocyte deformability is a nitric oxide - mediated factor in decreased capillary density during sepsis. Am J Physiol Heart Circ Physiol 2001;280(6):H2848-2856.

12. Bateman RM: C13 - pyruvate administration revealed differential metabolism between heart, liver and red blood cells and improved heart function during endotoxemia. Critical Care 2012;16(Suppl 1):P16.

13. Jagger JE, Bateman RM, Ellsworth ML, et al. Role of erythrocyte in regulating local $\mathrm{O}_{2}$ delivery mediated by hemoglobin oxygenation. Am J Physiol Heart Circ Physiol 2001;280(6): $\mathrm{H} 2833-2839$.

14. Larsen R, Gozzelino R, Jeney V, et al. A central role for free heme in the pathogenesis of severe sepsis. Sci Transl Med 2010;2(51):51ra71. 